Indicadores económicos que potencian la sostenibilidad turística de la ciudad de Riobamba

\title{
Indicadores económicos que potencian la sostenibilidad turística de la ciudad de Riobamba
}

\section{Economic indicators that make tourist sustainability in the city of Riobamba}

\section{Indicadores econômicos que aumentam a sustentabilidade do turismo na cidade de}

\section{Riobamba}

\author{
Catalina Margarita Verdugo Bernal ${ }^{\text {I }}$ \\ catalina.verdug@gmail.com \\ Cecilia Teresa Limaico Nieto II \\ cecilia.limaico@gmail.com \\ Mayra Elizabeth Cáceres Mena III \\ mayra19eli@hotmail.com \\ Jaime Giovany Bejar Suarez IV \\ jaime.bejar@gmail.com
}

Recibido: 13 de agosto de 2017 *Corregido: 28 de octubre de 2017 * Aceptado: 16 de noviembre de 2017

I. Magister en Turismo Sostenible y Desarrollo Local, Especialista Superior en Gestión Ambiental Mención Amazonia y Derechos Humanos, Master en Diseño, Gestión y Dirección de Proyectos, Ingeniera En Ecoturismo, Docente de la Escuela Politécnica de Chimborazo ESPOCH, Riobamba, Ecuador.

II. Magister en Educación Matemática, Doctora en Física, Licenciada en Ciencias de la Educación Profesora de Enseñanza Media en la Especialización de Matemática y Física, Docente de la Escuela Politécnica de Chimborazo ESPOCH, Riobamba, Ecuador.

III. Magister en Matemática Básica, Magister en Desarrollo de la Inteligencia y Educación, Doctora en Matemática, Docente de la Escuela Politécnica de Chimborazo ESPOCH, Riobamba, Ecuador.

Iv. Magister en Gestión Ambiental, Magister en Gerencia y Liderazgo Educacional, Diploma Superior de Cuarto Nivel en Pedagogías Innovadoras, Especialista en Gestión y Liderazgo Educativo, Doctor en Química Especialidad Físico Químico, Docente de la Escuela Politécnica de Chimborazo ESPOCH, Riobamba, Ecuador. 


\title{
Resumen
}

Se conoce que el turismo se ha convertido en una fuente clave de divisas y de empleo en las diferentes regiones, el sector genera más del 8\% de los trabajos en América Latina y casi el 13\% en el Caribe, superan los 79.000 millones de dólares. Varias son las ciudades que han demostrado tener un alto potencial turístico expresado en sus recursos naturales y culturales, logrando dinamizar la economía local de estos territorios y elevar el nivel de desarrollo en sus poblaciones (Banco de la República de Colombia, 2017, Boletin De Indicadores Económicos, 2017). En este contexto la ciudad de Riobamba, no es la excepción en encarrillarse en la actividad turística, pero se ha detectado la falta de información e indicadores actualizados y fiables, que sin duda alguna son los pilares en base a los cuales se deben tomar decisiones gestión pública y privada del sector turismo. Este estudio pretende analizar desde una perspectiva macro, sistemas de indicadores relacionados al ámbito económico influyentes en el turismo, para lograr su futura sostenibilidad. También se analizan indicadores planteados a nivel regional y finalmente se aterriza en indicadores aplicados en el ámbito local; todos estos provienen de fuentes de trayectoria comprobada como el World Bank, CEPAL, MINTUR, GADM Cuenca, entre otros. Adicional, se demuestra que un sistema de indicadores fiables puede incidir en la creación de políticas públicas que favorezcan a todos los actores turísticos y por ende mejoren la imagen de la ciudad y brinden oportunidades de pequeños negocios a sus habitantes. Es importante recalcar que este análisis es un aporte al macro-proyecto denominado Riobamba Sostenible que realizan conjuntamente el DITUR del GADM Riobamba y la Facultad de Recursos Naturales de la Escuela Superior Politécnica de Chimborazo.

Palabras clave: Indicadores; economía; sostenibilidad; turismo; Riobamba.

\begin{abstract}
It is known that tourism has become a key source of foreign currency and employment in different regions, the sector generates more than $8 \%$ of jobs in Latin America and almost $13 \%$ in the Caribbean, exceeding 79,000 million dollars. There are several cities that have demonstrated a high tourism potential expressed in their natural and cultural resources, achieving dynamism in the local economy of these territories and raising the level of development in their populations (Bank of the Republic of Colombia (2017, 03 3) Economic Indicators Bulletin 2017). In this context, the city of Riobamba is not the exception in getting behind the tourist activity, but it has been detected the lack of information and updated and reliable indicators, which undoubtedly are the pillars on which
\end{abstract}


Indicadores económicos que potencian la sostenibilidad turística de la ciudad de Riobamba

management decisions must be made public and private tourism sector. This study aims to analyze from a macro perspective systems of indicators related to the economic sphere influencing tourism, to achieve its future sustainability. It also analyzes indicators raised at the regional level and finally lands on indicators applied at the local level; all these come from sources of proven track record such as World Bank, ECLAC MINTUR, GADM Cuenca, among others. Additionally, it is shown that a system of reliable indicators can influence the creation of public policies that favor all tourism stakeholders and therefore improve the image of the city and provide small business opportunities to its inhabitants. It is important to emphasize that this analysis is a contribution to the macro-project called Riobamba Sostenible jointly carried out by the DITUR of GADM Riobamba and the Faculty of Natural Resources of the Polytechnic School of Chimborazo.

Keywords: Indicators; economy; sustainability; tourism; Riobamba.

\section{Resumo}

Sabe-se que o turismo se tornou uma das principais fontes de moeda estrangeira e emprego em diferentes regiões, o setor gera mais de 8\% dos empregos na América Latina e quase 13\% no Caribe, superior a 79.000 milhões de dólares. Há várias cidades que demonstraram um alto potencial turístico expresso em seus recursos naturais e culturais, alcançando dinamismo na economia local desses territórios e elevando o nível de desenvolvimento de suas populações (Banco da República da Colômbia, 2017, Boletim de Indicadores). Econômico, 2017). Neste contexto, a cidade de Riobamba não é a exceção para se apoiar na atividade turística, mas foi detectada a falta de informação e indicadores atualizados e confiáveis, que sem dúvida são os pilares sobre os quais as decisões gerenciais devem ser tomadas. sector do turismo público e privado. Este estudo tem como objetivo analisar, a partir de uma perspectiva macro, sistemas de indicadores relacionados à esfera econômica que são influentes no turismo, a fim de alcançar sua sustentabilidade futura. Também analisa os indicadores levantados no nível regional e, finalmente, chega aos indicadores aplicados no nível local; tudo isso vem de fontes de histórico comprovado, como Banco Mundial, CEPAL, MINTUR, GADM Cuenca, entre outros. Além disso, mostra-se que um sistema de indicadores confiáveis pode influenciar a criação de políticas públicas que favoreçam todos os interessados em turismo e, portanto, melhorem a imagem da cidade e ofereçam pequenas oportunidades de negócios aos seus habitantes. É importante ressaltar que esta análise é uma contribuição para o macro-projeto chamado 
Indicadores económicos que potencian la sostenibilidad turística de la ciudad de Riobamba

Riobamba Sostenible que é realizado conjuntamente pela DITUR do GADM Riobamba e pela Faculdade de Recursos Naturais da Escola Politécnica de Chimborazo.

Palavras chave: Indicadores; economia; sustentabilidade; turismo; Riobamba.

\section{Introducción}

Hoy el mundo sufre cambios constantes. En ese contexto, las empresas tanto públicas como privadas, deben buscar su desarrollo integral y competitivo, a través de la optimización de sus recursos y de la aplicación de estrategias que permitan el logro de objetivos empresariales.

Para ello, las organizaciones deben implementar estrategias que les permitan mejorar sus enfoques de aprendizaje, con la finalidad de desarrollar las competencias de sus recursos humanos a través del aprendizaje continuo.

En la actualidad todas las empresas necesitan que su recurso humano se involucre y se comprometa a resolver problemas sistémicos más rápidos que la competencia; por ende, se necesitan organizaciones inteligentes.

En el Ecuador, así como en otras regiones del mundo la Educación de Calidad se constituye en un reto ya que encamina sus esfuerzos hacia la formación de profesionales e investigadores de alta calidad académica; por lo que se ha establecido garantías que van de la mano con las mejores condiciones del ambiente, recursos, seguimiento de procesos y evaluación; con lo que se da la importancia al rol que tiene el estado, autoridades, docentes, estudiantes, y sociedad.

Por lo tanto, en una sociedad como la actual, caracterizada por altos niveles de: competencia, dinamismo, y complejidad, donde existen grandes stocks y flujos de información y conocimiento; el papel de la gestión del conocimiento al interior de los equipos directivos, no sólo puede ser un determinante estructural de las decisiones, sino que estas decisiones pueden incidir significativamente en el nivel de calidad que alcancen las universidades.

En este contexto, todas las universidades ecuatorianas compiten unas con otras, tanto las instituciones públicas como las privadas, haciendo notar que existe una brecha entre los roles que la sociedad del conocimiento demanda de las universidades y la calidad con la cual estas 
Indicadores económicos que potencian la sostenibilidad turística de la ciudad de Riobamba

organizaciones responden a dichas demandas. Sin duda, existe un vínculo ineludible entre los recursos y capacidades institucionales y los niveles de calidad alcanzados por las universidades.

Las Universidades Públicas de Manabí, Ecuador, no están ajenas a esta realidad. Sería de mucha utilidad estudiar el desarrollo y gestión del conocimiento en estas universidades para el logro de sus objetivos. Pero para ello es importante primeramente mostrar a través de la literatura publicada la relación entre la gestión del conocimiento y la gestión de la calidad.

\section{Metodología}

Para realizar este trabajo se hizo una búsqueda en el repositorio TDR para localizar tesis doctorales en red y en el directorio DOAJ para localizar artículos de revistas. Se utilizaron las palabras claves knowledge management AND quality management. Los documentos recuperados se evaluaron y se seleccionaron para este estudio 6 tesis y 4 artículos de revistas. Además, se utilizaron otros artículos de revistas que trataban la gestión del conocimiento y los documentos normalizativos de la ISO.

\section{Desarrollo}

El concepto de gestión del conocimiento no tiene definición única, sino que es explicado de diversas formas. Así Sánchez-Díaz (2006) plantea que a partir de la diferentes definiciones que aparecen en la literatura "la gestión del conocimiento es el procedimiento, estrategia, método, técnica, tarea, actividad, proceso, herramienta en conclusión que a nivel organizacional de manera colectiva, integradora, cíclica y sistemática; se va a encargar de encontrar, identificar, explotar, generar, adquirir, reunir, distribuir, tratar, estudiar, mejorar, proteger, aumentar, incrementar, diseminar, clasificar, revisar, conservar, almacenar, inventariar, administrar, coordinar, organizar, planificar, controlar, compartir, intercambiar, transferir, accesar, aprovechar, usar, utilizar; los flujos y recursos de conocimiento tanto disponibles como requeridos; con el propósito de que el conocimiento individual se transforme en colectivo, que no se pierda y se reutilice minimizando los recursos" (Sánchez-Díaz, y otros, 2006).

La Gestión del Conocimiento debe formular los objetivos del conocimiento, ubicar los activos del conocimiento y poseedores de conocimiento, identificar los tipos específicos de conocimiento tanto interno como externo, hacer visible el conocimiento interno, determinar los vacíos de conocimiento, identificar procesos que requieren un uso intensivo del conocimiento, buscar mecanismos para 
Indicadores económicos que potencian la sostenibilidad turística de la ciudad de Riobamba

aprovechar bases externas de conocimiento, determinar métodos para adquirir productos de conocimiento, desarrollar conocimiento, etc. La Gestión del Conocimiento crea valor con los activos intangibles de la organización.

Asimismo, se plantea que existen dos soportes básicos del conocimiento: El capital humano que interviene en los procesos de producción o de soporte organizacional (formación, capacidades, cualidades personales, entre otras); y, la información manejada en dichos procesos, que capacita a estas personas a incrementar su formación o habilidades para el desarrollo de sus tareas (Cordero Borjas, y otros, 2008).

En cuanto a la gestión de la calidad, se expresa que "son las actividades coordinadas para dirigir y controlar una organización en materia de calidad" (ISO, 2000).

La gestión de la calidad, según la norma internacional ISO 9004, se basa en ocho principios, desarrollados con la intención de que la alta dirección de las empresas los emplee para liderar la organización hacia un mejor desempeño. Ellos son: (ISO, 2000)

- Enfoque al cliente: Las organizaciones dependen de sus clientes y por lo tanto deberían comprender las necesidades actuales y futuras de los clientes, satisfacer los requisitos de los clientes y esforzarse en exceder las expectativas de los clientes.

- Liderazgo: Los líderes establecen la unidad de propósito y la orientación de la organización. Ellos deberían crear y mantener un ambiente interno, en el cual el personal pueda llegar a involucrarse totalmente en el logro de los objetivos de la organización.

- Participación del personal: El personal, a todos los niveles, es la esencia de una organización y su total compromiso posibilita que sus habilidades sean usadas para el beneficio de la organización.

- Enfoque basado en procesos: Un resultado deseado se alcanza más eficientemente cuando las actividades y los recursos relacionados se gestionan como un proceso.

- Enfoque de sistema para la gestión: Identificar, entender y gestionar los procesos interrelacionados como un sistema, contribuye a la eficacia y eficiencia de una organización en el logro de sus objetivos.

- Mejora continua: La mejora continua del desempeño global de la organización debería ser un objetivo permanente de ésta. 
- Enfoque basado en hechos para la toma de decisión: Las decisiones eficaces se basan en el análisis de los datos y la información.

- Relaciones mutuamente beneficiosas con el proveedor: Una organización y sus proveedores son interdependientes, y una relación mutuamente beneficiosa aumenta la capacidad de ambos para crear valor.

La relación entre la gestión del conocimiento y la gestión de calidad se evidencia en los siguientes trabajos:

Olmedo (2011) estudia la evolución de los modelos de calidad total hacia modelos de gestión del conocimiento. "La definición del proceso de gestión del conocimiento establece los parámetros básicos en los que se sustenta un sistema de gestión de la calidad, que a su vez este último establece la estructura organizativa en función del proceso de gestión del conocimiento. Un sistema de información que automatiza los flujos de información y la redefinición de una cultura organizacional orientada al conocimiento es el marco de trabajo del modelo EFQM. Para su implementación es necesario: establecer las etapas del proceso de gestión del conocimiento de la organización, una gestión por procesos (sistema de gestión de la calidad), un sistema de gestión de la información y su sistema de gestión de las personas" (Olmedo Narbona, 2011).

Garzón (2012) describe e interpreta los efectos de la implementación del proyecto de calidad y mejora continua (PQiMC) sobre el proceso de enseñanza aprendizaje, en siete centros de formación profesional reglada en Cataluña. "Se puede afirmar que los procesos de mejora continua en los centros educativos tienen efecto sobre el proceso de enseñanza-aprendizaje, si las acciones de mejora se realizan de manera sistemática y planeada por medio de la adopción de un modelo de gestión de la calidad que sea coherente con el contexto externo, interno y del aula, del centro o grupo de centros, que guíe los procesos y procedimientos por medio de la definición de objetivos y acciones concretas, que contemple espacios de reflexión y motivación para la participación activa de todos los miembros de la comunidad educativa, que su implementación esté liderada por el equipo directivo del centro educativo, que contenga lineamientos generales e instrumentos para la obtención de la información, que motive la formación de los gestores de calidad en este campo y la formación continua de los profesores en el ámbito pedagógico y/o técnico, y, que motive el trabajo en red de 
Indicadores económicos que potencian la sostenibilidad turística de la ciudad de Riobamba

centros para facilitar la puesta en común de buenas prácticas y un espacio común de reflexión" (Garzón Castrillon, 2012).

Rodríguez (2012), explora la naturaleza de la vinculación existente entre el proceso de gestión del conocimiento en los equipos de alta dirección de las universidades y la calidad lograda por estas instituciones de educación superior. El estudio demostró que "Los estilos de liderazgo y la cultura organizacional son los determinantes del proceso de gestión del conocimiento; la Gestión del conocimiento impacta sobre la calidad del quehacer académico, mismo que configura la calidad institucional; y, los estilos de liderazgo participativo y colaborativo que determinan la cultura organizacional de innovación y comunitaria, la que permite crear, compartir conocimiento y procesos esenciales para aplicar conocimiento" (Rodríguez Ponce, 2012).

La investigación de Porras (2013) tuvo como objetivo identificar las variables de tipo administrativo, organizacional, cultural y de conocimientos sobre gestión de la calidad, que condicionan la implantación del Sistema de Gestión de la Calidad (SGC) en las instituciones públicas de educación preescolar, básica y media, del Municipio de Villavicencio (Colombia). "Para asegurar el éxito de la implantación del SGC los colegios deben prestar atención a los distintos componentes que obtuvieron menores valoraciones, priorizándolos según su nivel de urgencia, tendencia e impacto. Habrá algunos que a corto plazo pueden ser resueltos, otros a mediano y largo plazo, pero ante todo, elaborar el plan de mejoramiento basándose en los resultados de la autoevaluación institucional, e implementar los mecanismos adecuados para su seguimiento, tales como la detección y control de no conformidades, mediante auditorías internas periódicas y oportunas, siempre en el marco de la participación democrática, y la auto implicación de toda la comunidad educativa; y, por otra parte, las Secretarías de Educación y el Ministerio de Educación Nacional, deben formular las directrices que guíen u orienten a los colegios públicos de preescolar, básica y media, bajo su administración, en la implantación del SGC, para asegurar el éxito de dicho proceso, aún en gestación en el país; para ello, ponemos a su disposición, el Plan de Gestión Integral de la Calidad (PGIC), configurado a partir de la experiencia adquirida en el desarrollo de las distintas etapas de esta investigación, adelantada durante tres años (Porras Aguirre, 2013).

Franch (2014), diagnostica en qué medida, la gestión del conocimiento puede constituir una herramienta de apoyo en los procesos de decisiones. "(...) Con relación a los modelos para el 
Indicadores económicos que potencian la sostenibilidad turística de la ciudad de Riobamba

desarrollo de la gestión del conocimiento, se puede observar que existen infinidades de ellos que de forma simplificada y esquemática representan el fenómeno, delimitan sus dimensiones, permiten una visión aproximada de los objetivos que persiguen, describen su estructura, orientan estrategias y precisan aspectos relevantes para su adecuada comprensión, sin embargo, no se identifican diseños asociados al mejoramiento de la TD basado en la GC, constituyendo esto una brecha desde el punto de vista investigativo" (Franch León, 2014).

Fernández Jeri (2014) evalúa los agentes facilitadores para la mejora de la calidad en la Universidad Nacional Mayor de San Marcos (UNMSM), Universidad Nacional de Ingeniería(UNI) y Universidad Nacional Agraria La Molina (UNALM). "Aplicando el formato de la Fundación Europea (EFQM) para evaluar los agentes facilitadores para la calidad en la UNMSM, UNI, UNALM, se encuentra que las universidades en su conjunto, están preparadas para desarrollar un sistema de gestión de la calidad, pero se deben ajustar al contexto y posibilidad; se encuentra que dos agentes facilitadores para la calidad en las 3 universidades deben mejorarse y son los procesos y la existencia de recursos y alianzas; y, también otro factor importante para mejorar la calidad es el liderazgo, respecto al cual se encontró que en las universidades si existe cierto tipo de liderazgo en las autoridades. Tanto en la UNMSM como en la UNALM se describe un liderazgo a través de que 'los líderes se implican personalmente para garantizar el desarrollo, implantación y mejora continua del sistema de gestión de la universidad'. Y en el caso de la UNI: 'Los líderes desarrollan la misión, visión, valores y principios; así como lideran en su relación con clientes, partners y representantes de la sociedad'.

Liberona y otros (2013), consideran que la gestión del conocimiento es una nueva disciplina que facilita la creación, el almacenamiento, la transferencia y la aplicación de conocimientos en las organizaciones. Este trabajo explora el estado general de la implantación de estos programas en Chile y cuáles son las principales dificultades para desarrollarlos. Se identificaron los siguientes problemas principales:

- Las empresas cuentan con una base conceptual limitada sobre el conocimiento y su proceso de gestión.

- No tienen un enfoque sistemático sobre cómo apropiarse y compartir el conocimiento. 
Indicadores económicos que potencian la sostenibilidad turística de la ciudad de Riobamba

- Desconocen los beneficios de la gestión del conocimiento y suelen concentrarse en resolver problemáticas puntuales a corto plazo en vez de mejorar la eficiencia del aprendizaje organizacional, su correcta utilización y su desarrollo.

- Las empresas desconocen cuánto capital intelectual tienen y cuánto pierden, van resolviendo los problemas relacionados con el conocimiento mediante soluciones a corto plazo, como el volver a reclutar, entrenar y reentrenar permanentemente, o generar manuales de procedimientos que suelen estar desactualizados y no son consultados.

"Es necesario lograr que la organización en su conjunto participe activamente y colabore en el desarrollo de las soluciones y prácticas de gestión del conocimiento (algunas de las prácticas colaborativas más utilizadas son wikis, comunidades de práctica, guías de expertos y herramientas de colaboración). Es fundamental que los colaboradores en la organización quieran compartir voluntariamente su conocimiento y estén comprometidos con el programa; existe una serie de prácticas que incentivan esta colaboración, como lo son el construir relaciones entre los trabajadores para que se conozcan y compartan diferentes situaciones, entreguen mensajes y logren objetivos relacionados con el hecho de compartir el conocimiento, desarrollen espacios físicos que incentiven el encuentro y comunicación y definan procesos para compartir conocimiento dentro de los flujos de trabajo; y, los responsables de los programas de la gestión del conocimiento deben convertirse en emprendedores al interior de la organización y ser obsesivos acerca de cómo capturar, distribuir y aplicar viejos conocimientos para poder crear nuevos conocimientos e innovar ya que cada vez más el éxito empresarial dependerá de las habilidades para cambiar y adaptarse; a su vez, un programa de gestión del conocimiento bien implementado colabora de manera eficaz con el desarrollo de estas habilidades" (Liberona, y otros, 2013).

Saldarriaga (2013) aborda la responsabilidad social y la gestión del conocimiento como estrategias de gestión humana, conexas e inseparables, en las organizaciones. Estas estrategias hacen posible que la organización mire hacia sí misma y reconozca los impactos que estas prácticas tienen sobre sus miembros, asumiéndolas no solo exclusivamente como actividades que le generan ganancias económicas y visibilidad en el escenario social, sino como parte integral de su forma de concebir y administrar su talento humano. 
"Puede afirmarse tendencias relacionadas con la responsabilidad social y la gestión del conocimiento son ampliamente utilizadas por las empresas colombianas, pero en muchas de ellas no se encuentran articuladas a la estrategia de gestión humana. El uso e implementación en las empresas colombianas de las actuales tendencias de gestión humana, entre ellas, la responsabilidad social y la gestión del conocimiento, evidencia un creciente interés por encontrar alternativas que les permitan ser más productivas y competitivas, valiéndose de estrategias que cada vez son más utilizadas en el ámbito internacional; lo que resulta interesante es que dichas tendencias y estrategias en el contexto nacional parecen no responder a un direccionamiento estratégico del proceso de gestión humana, alineado a la estrategia del negocio, sino a acciones de carácter puntual de las que se esperan beneficios rápidos y no relacionados con el desarrollo de las personas de la organización (Saldarriaga Ríos, 2013).

Silas (2014) aborda el tema de la calidad en la educación superior y su relación con los esquemas nacionales para la acreditación de instituciones y planes y programas educativos. Se presentan datos de las prácticas en otras regiones del mundo, especialmente los países sajones y algunos asiáticos para, posteriormente, elaborar una breve descripción de los mecanismos latinoamericanos para la acreditación de la calidad. El autor expone dos ideas:

a. A pesar de que la retórica está más presente que las buenas prácticas institucionales, los actores involucrados en la provisión de educación superior y en su regulación y acreditación han dado pasos importantes en la mejora cualitativa de la formación terciaria latinoamericana y

b. Los mecanismos revisados se centran más en esquemas que aseguren la eficiencia de acuerdo con estándares internacionales, lo que deja de lado la equidad como aspecto fundamental de la función educativa.

"Los esfuerzos para valorar y acreditar la calidad de las instituciones de ES y la formación que ofrecen han tenido un crecimiento notable en las dos últimas décadas y se ha dejado sentir prácticamente en todos los continentes; el incremento en el número de esfuerzos nacionales e incluso regionales, agencias acreditadoras, instituciones y programas que han sido acreditados y, especialmente en la producción de documentos académicos, ha sido igualmente notorio. La realización de foros, simposios y congresos sobre la materia no deja duda de su visibilidad. Los mecanismos que siguen las agencias acreditadoras guardan una gran similitud, pues valoran 
Indicadores económicos que potencian la sostenibilidad turística de la ciudad de Riobamba

prácticamente los mismos aspectos y solamente algunos casos, como el malayo, van más allá de lo usual. En América Latina, el énfasis en la acreditación de la calidad presenta una perspectiva centrada en la eficiencia que parece apegarse más a esquemas pragmáticos que inducen al isomorfismo debido a las ineludibles condiciones socioeconómicas y políticas que enfrentan los sistemas de ES. En este sentido es notorio el énfasis de los gobiernos nacionales en redoblar esfuerzos encaminados al logro de políticas de acreditación válidas, por lo que es presumible que seguirán vigentes en el corto y largo plazos. Por otro lado, sin soslayar la importancia de los mecanismos acreditadores, es relevante señalar dos fenómenos tangenciales que parecen estar desarrollándose. El primero es la creación de una industria de la evaluación y acreditación que ha permitido que existan agencias privadas de acreditación o incluso algunos organismos que se centran en auxiliar a las instituciones en "salir aprobadas" en el proceso que, de manera homóloga a las IES con fines de lucro, realizan operaciones valoradas por el sistema educativo con una lógica comercial. El segundo parece mostrar cómo los aspectos evaluados y los mecanismos de acreditación se centran más en esquemas que aseguren la eficiencia institucional de acuerdo con estándares internacionales y que parecen ignorar un tema toral como es el de la equidad (SILAS Casillas, 2014).

Espinosa (2015) caracteriza los principales rasgos de los sistemas de aseguramiento de la calidad en la educación superior implementados en las últimas dos décadas en América Latina, junto con la delimitación de los logros obtenidos a la fecha y los desafíos que están enfrentado dichos sistemas.

"(...) los avances que se han ido registrando con distintos énfasis en los países de la región, dejan de manifiesto que el aseguramiento de la calidad de la docencia y de la gestión, tanto a nivel de instituciones como de carreras de pregrado y programas de posgrado en el nivel terciario, es una realidad. En efecto, se han ido consolidando paulatinamente, al interior de las universidades, las unidades de análisis institucional y las direcciones de planificación estratégica, cuestión que era impensable veinte años atrás. En forma simultánea, se han ido instalando de manera progresiva mecanismos y procedimientos de control y seguimiento de procesos, que han permitido que las instituciones puedan autoevaluar su gestión en diferentes niveles: docencia, investigación, extensión, vinculación con el medio. A su vez, se han ido consolidando las agencias de acreditación (tanto las estatales como las privadas), en un proceso que no ha estado exento de dificultades por todo lo que implica. Se está experimentando un proceso de aprendizaje y crecimiento continuo, tanto a nivel de 
Indicadores económicos que potencian la sostenibilidad turística de la ciudad de Riobamba

agencias acreditadoras como de las propias instituciones de educación superior. (ESPINOZA Díaz, 2015)

\section{Conclusiones}

El término gestión del conocimiento responde a multitud de definiciones. Hay quienes se centran en las personas, su desarrollo y el aprendizaje, otros en la gestión de información y su almacenamiento, y algunos en la medición del Capital Intelectual. Por lo tanto existen elementos comunes entre la gestión del conocimiento y la gestión de la calidad.

La gestión del conocimiento contribuye a la materialización de todos los principios de la gestión de la calidad, todos necesitan del aprendizaje organizacional que no se logra sin una adecuada gestión del conocimiento. ES decir, la implementación de la gestión del conocimiento puede contribuir a incrementar la eficacia y eficiencia de la gestión de la calidad. El Modelo EFQM de Excelencia ha sido utilizado por varios autores ya que integra la gestión del conocimiento y la gestión de la calidad.

La gestión del conocimiento y la gestión de la calidad en la universidad deben articularse de manera tal que en los objetivos de calidad se incluyan los objetivos de la gestión del conocimiento y viceversa. La universidad debe lograr en los educandos el pensamiento crítico, independiente, la innovación y el aprendizaje a lo largo de la vida; en esencia un proceso de enseñanza-aprendizaje de calidad.

Este trabajo permite hacer una investigación aplicativa de corte cuali-cuantitativo sobre la realidad de las Universidades Públicas que determine como contribuye la implementación de un modelo explicativo de gestión del conocimiento en los equipos directivos de las universidades públicas de Manabí en el aseguramiento de la calidad institucional.

\section{Referencias bibliográficas}

Cordero Borjas, A. E. y García Fernández, F. 2008. Knowledge Management and Work Teams: Observatorio Laboral. Revista Venezolana. 2008.

ESPINOZA Díaz, Ó. 2015. Los sistemas de aseguramiento de la calidad en la educación superior en América Latina. ResearchGate. 2015, 29. 
Indicadores económicos que potencian la sostenibilidad turística de la ciudad de Riobamba

FERNANDEZ JERI, L. H. 2014. Evaluación de los agentes facilitadores Para la mejora de la calidad en la Universidad Nacional Mayor de San Marcos, Universidad Nacional de Ingeniería y Universidad Nacional Agraria la Molina. s.l. : Tesis para optar por el Título de Doctor, 2014.

FRANCH LEÓN, K. 2014. La gestión del conocimiento como herramienta de apoyo al proceso de decisión en las organizaciones. El caso de las organizaciones cubanas. UNIVERSIDAD DE SANTIAGO DE COMPOSTELA. Facultad de Ciencias políticas y Sociales: Tesis para optar por el Título de Doctor en Ciencias, 2014.

GARZON CASTRILLON, AJ-. 2012. La mejora continua y la calidad en instituciones de formación profesional. El proceso de enseñanza-aprendizaje. UNIVERSIDAD AUTÓNOMA DE BARCELONA. Facultad de Ciencias de la Educación.: Tesis para optar el grado académico de Doctor, 2012.

GEOFFROY Pitta, E. 2013. Estudio del sistema de aseguramiento de la calidad de la Educación Superior. Un análisis politológico de formulación de política pública. Revista Enfoques: Ciencia Política y Administración Pública. 2013, Vol. XI, 19.

INCHE MITMA, JL. 2010. Modelo Dinámico de Gestión del Conocimiento basado en el Aprendizaje Organizacional en una Institución Educativa en el Perú. UNIVERSIDAD NACIONAL MAYOR DE SAN MARCOS Facultad de Ciencias Administrativas: Tesis, 2010.

LIBERONA, D. y RUIZ, M. 2013. Análisis de la implementación de programas de gestión del conocimiento en empresas chilenas. Estudios Gerenciales. 2013, Vol. 29, 127.

SALDARRIAGA Ríos, J.G. 2013. Responsabilidad social y gestión del conocimiento como estrategias de gestión humana. Estudios Gerenciales. 2013, Vol. 29, 1.

Sánchez-Díaz, M. y Vega Valdés, J.C. 2006. La gestión del conocimiento y su relación con otras gestiones. Ciencias de la Información. 2006, Vol. 37, 2-3.

SILAS Casillas, J. C. 2014. Calidad y Acreditación en la Educación Superior: Realidades y retos para América Latina. Páginas de Educación. 2014, Vol. 7, 2. 